\title{
ON THE STRUCTURE OF ALGEBRAS WITH NONZERO RADICAL
}

\author{
G. HOCHSCHILD
}

Introduction. If one attempts to make a systematic study of algebras with nonzero radical one soon realizes that the main difficulty is that of singling out a set of structural characteristics which could constitute a suitable center of interest in a general theory. In the study of simple and semisimple algebras, the full matrix algebras over the groundfield serve as models for the "perfect" structure, and the greatest departure from this which one has to consider consists in the ground field being replaced by a division algebra. On the other hand, even among the nilpotent algebras, it would be difficult to decide what type is to be regarded as representing the "perfect" structure.

The first type that one might think of in this connection is the following: There is a single element, $x$, with $x^{n+1}=0$, for some positive integer $n$, whose powers $x, x^{2}, \cdots, x^{n}$ constitute a linear basis for the algebra. However, this is evidently far too special a type to serve as a standard. A natural generalization of this, which turns out to be more suitable, is obtained by replacing the one-dimensional subspace, $(x)$, by a subspace of arbitrary dimension. With suitable additional requirements in the case of non-nilpotent algebras, we are led to the notion of a "quasicyclic" algebra whose radical has a considerably more transparent structure than a general nilpotent algebra.

We also introduce a more restrictive notion, that of a "maximal" algebra, and it will be shown that every algebra whose quotient by the radical is separable or $\{0\}$ is the homomorphic image of a "related" maximal (quasicyclic) algebra. Since the structure of maximal algebras is determined completely by separable algebras and their representations our structure theory decomposes into two parts: The study of two-sided ideals contained in the radical of a maximal algebra, and the study of separable algebras and their representations.

Throughout this paper we shall deal only with algebras whose quotients by the radical are either $\{0\}$ or separable. In particular, this will be the case for all algebras over a perfect field. It is to be noted that in this approach there is no need for a general representation theory of non-semisimple algebras.

1. Related algebras. We consider algebras $B$, with radical $R$, such that $B / R$ is separable or $\{0\}$. Two such algebras, $B_{1}$ and $B_{2}$, are said

Received by the editors October 24, 1946. 
to be related if:

(1) $B_{1} / R_{1}^{2} \approx B_{2} / R_{2}^{2}$.

(2) The indices of nilpotency of $R_{1}$ and $R_{2}$ are the same.

By the index of nilpotency of a nilpotent algebra, $R$, we shall mean the least non-negative integer $k$ for which $R^{k+1}=\{0\}$.

By Wedderburn's structure theorem, ${ }^{1}$ we know that $B$ contains a subalgebra, $A$, which is mapped isomorphically onto $B / R$ by the natural homomorphism of $B$ onto $B / R$. Then-as a vector space- $B$ is the direct sum of $A$ and $R$, and for any such decomposition the multiplication in $B$ induces the structure of a two-sided $B / R$-module ${ }^{2}$ in $B$. Moreover, $R^{2}$ is a submodule of $R$, and the induced $B / R$-module structure in $R / R^{2}$ is determined uniquely by $B$, or even by $B / R^{2}$. Related algebras determine isomorphic modules $R / R^{2}$. If we denote by $T$ a model for the $A$-module $R / R^{2}$ and write the linear transformations in $T$ which correspond to an element $a \in A$ as $t \rightarrow a \cdot t$ and $t \rightarrow t \cdot a$, we obtain a model for $B / R^{2}$ whose underlying vector space is the direct sum $(A, T)$ of $A$ and $T$, and where multiplication is defined by the formula

$$
\left(a_{1}, t_{1}\right)\left(a_{2}, t_{2}\right)=\left(a_{1} a_{2}, a_{1} \cdot t_{2}+t_{1} \cdot a_{2}\right) .
$$

For, if $\bar{\sigma}$ is an operator isomorphism of $T$ onto $R / R^{2}$, the mapping $(a, t) \rightarrow \bar{a}+\bar{\sigma}\{t\}$, where $\bar{a}$ is the coset $\bmod R^{2}$ of $a$ in $B / R^{2}$, is evidently an isomorphism of this algebra onto $B / R^{2}$.

Later, we shall construct a maximal algebra related to $B$ of which $B$ is a homomorphic image by suitably "enlarging" the radical $(0, T)$ of this algebra. For this construction we require a few auxiliary notions and a decomposition theorem for two-sided modules. These will be given in the next two sections.

2. $A$-modules. Let $A$ be an algebra over the field $F, T$ a vector space over $F$. Let $\sigma$ be a homomorphism of $A$ into the algebra of linear transformations of $T, \sigma^{*}$ an antihomomorphism of $A$ into this algebra. If, for all $a_{i} \in A$, we have $\sigma\left\{a_{1}\right\} \sigma^{*}\left\{a_{2}\right\}=\sigma^{*}\left\{a_{2}\right\} \sigma\left\{a_{1}\right\}$ we say that the set $\left(A, T, \sigma, \sigma^{*}\right)$, or, simply, $T$ is a (two-sided) $A$-module. If we write $(\sigma\{a\})\{t\}=a \cdot t,\left(\sigma^{*}\{a\}\right)\{t\}=t \cdot a$, we may express our conditions by saying that the operations satisfy all the formal requirements for a multiplication. We define submodules and quotient modules in the usual manner. A module $M$ is said to be semisimple if to every submodule $K$ we can find a complementary submodule $L$ such that $M$ is the direct sum of $K$ and $L . M$ is called simple if $\{0\}$

${ }^{1}$ See, for instance, N. Jacobson, The theory of rings, Mathematical Surveys, vol. 2, chap. $5, \S 20$.

2 Cf. $\$ 2$ of this paper. 
and $M$ are its only submodules. We shall require the following theorem:

Theorem 2.1. If $A$ is separable then every two-sided $A$-module is semisimple.

This is an easy generalization of the well known corresponding theorem concerning semisimple algebras and their representations, that is, their left modules. ${ }^{3}$ In fact, denote by $A^{*}$ the algebra obtained by adjoining a new identity element, 1 , to $A$. Let $e$ be the identity element of $A$. Then we have $(1-e) A=\{0\}=A(1-e)$, and $(1-e)^{2}=1-e$, whence we see that $A^{*}$ is the direct sum of $A$ and the ground field $F$. Hence $A^{*}$ is separable. Now let $\tilde{A}^{*}$ be the algebra inversely isomorphic with $A^{*}$. Then $\tilde{A}^{*}$ is evidently separable also, and hence, by well known results, ${ }^{4}$ the Kronecker product $A^{*} \times \tilde{A}^{*}$ is separable, or, in particular, semisimple. Hence every left $A^{*} \times \tilde{A}^{*}$-module is semisimple. Now if $M$ is any $A$-module we can define in $M$ the structure of a left $A^{*} \times \tilde{A}^{*}$ module by setting $1 \cdot m=m=m \cdot 1$, for every $m \in M$, and $\left(a_{1}^{*} \times \tilde{a}_{2}^{*}\right) \cdot m=a_{1}^{*} \cdot m \cdot a_{2}^{*}$. The statement that this left $A^{*} \times \tilde{A}^{*}$ module is semisimple is then evidently equivalent to the statement that the given two sided $A$-module $M$ is semisimple. (Because of the 1 , there is a $1-1$ correspondence between two-sided submodules and one-sided submodules of $M$.)

3. Remarks on Kronecker products. It will be convenient to review some basic facts concerning Kronecker products of vector spaces. Let $L_{1}, \cdots, L_{n}$ be a set of (finite-dimensional) vector spaces over a field $F$. We denote by $M\left(L_{1}, \cdots, L_{n}\right)$ the vector space formed by the $n$-linear functions on the direct sum of these spaces, that is, by the functions $f$ mapping $\left(L_{1}, \cdots, L_{n}\right)$ into $F$ in such a way that $f$ is linear on each $L_{i}$ when the coordinates in the remaining $L_{i}$ are kept fixed. By the Kronecker product, $L_{1} \times \cdots \times L_{n}$, of the $L_{i}$ is meant the vector space dual to $M\left(L_{1}, \cdots, L_{n}\right)$, that is, the vector space whose elements are the linear mappings of $M\left(L_{1}, \cdots, L_{n}\right)$ into $F$. If $\left(z_{1}, \cdots, z_{n}\right) \in\left(L_{1}, \cdots, L_{n}\right)$ we obtain an element $z_{1} \times \cdots \times z_{n}$ in $L_{1} \times \cdots \times L_{n}$ by setting $\left(z_{1} \times \cdots \times z_{n}\right)\{f\}$ $=f\left\{z_{1}, \cdots, z_{n}\right\}$, for every $f \in M\left(L_{1}, \cdots, L_{n}\right)$. Evidently, the mapping $\left(z_{1}, \cdots, z_{n}\right) \rightarrow z_{1} \times \cdots \times z_{n}$ is $n$-linear. Moreover, the images $z_{1} \times \cdots \times z_{n}$ span $L_{1} \times \cdots \times L_{n}$ over $F$. It follows that the mapping $\left(z_{1} \times \cdots \times z_{k}, z_{k+1} \times \cdots \times z_{l}\right) \rightarrow z_{1} \times \cdots \times z_{l}$ induces a bilinear mapping of $\left(L_{1} \times \cdots \times L_{k}, L_{k+1} \times \cdots \times L_{l}\right)$ into $L_{1} \times \cdots \times L_{l}$.

${ }^{3}$ N. Jacobson, loc. cit. chap. $4, \$ 12$.

${ }^{4}$ See van der Waerden, Moderne Algebra, 1st ed., vol. 2, \$119. 
Moreover, this mapping is distributive. If $u \in L_{1} \times \cdots \times L_{k}$, and $v \in L_{k+1} \times \cdots \times L_{l}$, we shall denote the image of $(u, v)$ under the above mapping by $u \times v$. Then it is easy to see that $(u \times v) \times w$ $=u \times(v \times w)$. Thus the mapping $(u, v) \rightarrow u \times v$ behaves like a multiplication. Indeed, we shall use this operation later in order to define a multiplication in a vector space which is the direct sum of Kronecker products.

4. Maximal related extensions and quasicyclic algebras. Let $B$ be an algebra, $R$ the radical of $B$. We say that $B$ is quasicyclic if the following conditions are satisfied:

(1) $B$ contains a subalgebra $A$ which is mapped isomorphically onto $B / R$ by the natural homomorphism of $B$ onto $B / R$, so that $R$ becomes an $A$-module in the natural fashion.

(2) $R$, as an $A$-module (two-sided), admits of a direct decomposition into submodules $R_{1}, \cdots, R_{n}$, where $R_{k}$ is the ordinary $k$ th power of $R_{1}$, and $n$ is the index of nilpotency of $R$.

If $B$ is an algebra and $\beta$ is a homomorphism of $B$ onto an algebra $A$, the pair $(B, \beta)$ is called an extension of $A .(B, \beta)$ is called a related extension of $A$ if $B$ and $A$ are related. $A$ is called maximal if, in every related extension $(B, \beta)$ of $A, \beta$ is an isomorphism. A related extension $(B, \beta)$ of $A$ is called maximal if $B$ is maximal.

Our main result is the following:

THEOREM 4.1. Let $B$ be an algebra with radical $R$ such that $B / R$ is separable or $\{0\}$. Then there exists a maximal related extension $(C, \gamma)$ of $B$, such that $C$ is quasicyclic. If $\left(C_{i}, \gamma_{i}\right)$ is a maximal related extension of $B_{i}, i=1,2$, and if $B_{1}$ and $B_{2}$ are related, then $C_{1}$ and $C_{2}$ are isomorphic.

Proof. Let $A$ be a subalgebra of $B$ which is mapped isomorphically onto $B / R$ by the natural homomorphism of $B$ onto $B / R$. Now consider the $A$-module $R$, and the submodule $R^{2}$. By Theorem 2.1, there exists a complementary submodule $T$ such that $R$, as an $A$-module, is the direct sum of $T$ and $R^{2}$. Let $n$ be the least integer $k$ such that $R^{k+1}=\{0\}$. (We may assume $n \geqq 1$.) We form the Kronecker products $T^{(k)}$ of $T$ by itself $k$ times, $k=1, \cdots, n$. We can make each $T^{(k)}$ into an $A$-module by setting $a \cdot\left(t_{1} \times \cdots \times t_{k}\right)=a t_{1} \times \cdots \times t_{k}$, and $\left(t_{1} \times \cdots \times t_{k}\right) \cdot a=t_{1} \times \cdots \times t_{k} a$, where $a t_{1}$ and $t_{k} a$ stand for the products in $B$, which lie in $T$, by our construction of $T$. We consider the direct sum, $S$, of these $T^{(k)}$. There is a unique linear mapping $\sigma$ of $S$ into $R$ such that $\sigma\left\{t_{1} \times \cdots \times t_{k}\right\}=t_{1} \cdots t_{k}$. Evidently, $\sigma$ is an operator homomorphism of the $A$-module $S$ onto the $A$-module $R$. The fact that $\sigma$ is onto follows from the fact that every $r \in R$ can be 
written as a sum of products of elements in $T$. Now we make $S$ into a nilpotent algebra related to $R$ by means of the Kronecker multiplication discussed in $\S 3$. More precisely, for $S_{k_{1}} \in T^{\left(k_{1}\right)}$ and $S_{k_{2}} \in T^{\left(k_{2}\right)}$, we set $S_{k_{1}} * S_{k_{2}}=S_{k_{1}} \times S_{k_{2}}$, when $k_{1}+k_{2} \leqq n$, and 0 otherwise. Then $\sigma$ is evidently a homomorphism of the algebra $S$ onto the algebra $R$. This still requires modification :

Let $U_{2}$ be the subspace of $T^{(2)}$ which is spanned by the elements of the form $t_{1} a \times t_{2}-t_{1} \times a t_{2}$, with $a \in A, t_{1}, t_{2} \in T$. If $U_{k}$ is already defined as a subspace of $T^{(k)}$ we define $U_{k+1}$ as the subspace of $T^{(k+1)}$ which is spanned by $T \times U_{k}$ and $U_{k} \times T$. Evidently, each $U_{k}$ is a submodule of $T^{(k)}$, and is contained in the kernel of $\sigma$. Now we can choose a submodule $V_{k}$ of $T^{(k)}$ which is complementary to $U_{k}$. Denote the projection of $T^{(k)}$ onto $V_{k}$ by $\pi_{k}$. Write $T=V_{1}$, and let $E=V_{1}+\cdots+V_{n}$ (this sum is direct). We define a multiplication in $E$ by setting, for $z_{k} \in V_{k}, z_{i} \circ z_{j}=\pi_{i+j}\left\{z_{i} \times z_{j}\right\}$, when $i+j \leqq n$, and 0 otherwise. This is evidently bilinear. Moreover, since $\pi_{i+j}\left\{z_{i} \times z_{j}\right\} \times z_{k}-z_{i} \times \pi_{j+k}$ - $\left\{z_{j} \times z_{k}\right\} \in U_{i+j+k}$ when $i+j+k \leqq n$, we have always $\left(z_{i} \circ z_{j}\right) \circ z_{k}$ $=z_{i} \circ\left(z_{j} \circ z_{k}\right)$. Thus $E$ has the structure of a nilpotent algebra. The mapping $\sigma$ induces a homomorphism of the algebra $E$ onto the algebra $R$; in fact, $\sigma\left\{z_{i} \circ z_{j}\right\}=\sigma\left\{z_{i} * z_{j}\right\}$. Since the index of nilpotency of $E$ is not greater than $n$, by construction, and since $\sigma\left\{E^{n}\right\}$ $=R^{n}$, it follows that the index of nilpotency of $E$ is exactly $n$. Evidently, $E / E^{2} \approx T \approx R / R^{2}$, whence $E$ is related to $R$. Finally, $\sigma$ induces an operator homomorphism of the $A$-module $E$ onto the $A$-module $R$.

Now we construct an algebra, $C$, out of the direct sum $(A, E)$ of the vector spaces of $A$ and $E$ by setting

$$
\left(a_{1}, e_{1}\right)\left(a_{2}, e_{2}\right)=\left(a_{1} a_{2}, a_{1} \cdot e_{2}+e_{1} \cdot a_{2}+e_{1} \circ e_{2}\right) .
$$

Since $E$ is an $A$-module and since, evidently, $a \cdot\left(e_{1} \circ e_{2}\right)=\left(a \cdot e_{1}\right) \circ e_{2}$, and $\left(e_{1} \circ e_{2}\right) \cdot a=e_{1} \circ\left(e_{2} \cdot a\right)$, the associativity condition reduces to $\left(e_{1} \cdot a\right) \circ e_{2}=e_{1} \circ\left(a \cdot e_{2}\right)$. This is satisfied in virtue of our definitions for the $U_{k}$ and $\pi_{k}$. If we define $\gamma\{(a, e)\}=a+\sigma\{e\}$, we see immediately that $\gamma$ is a homomorphism of $C$ onto $B$. Moreover, $\gamma$ induces an isomorphism of $C / E^{2} \approx(A, T)$ onto $B / R^{2}$. Hence $C$ is related to $B$. Evidently, $C$ is quasicyclic. For convenience of reference, let us call a related extension $(C, \gamma)$ of $B$, which is obtained by a construction such as we have just described, a standard related extension of $B$.

Now let $B_{1}$ and $B_{2}$ be any two related algebras with radicals $R_{1}$ and $R_{2}$, respectively. Let $\rho$ be an isomorphism of $B_{1} / R_{1}^{2}$ onto $B_{2} / R_{2}^{2}$. Let $\left(C_{1}, \gamma_{1}\right)$ and $\left(C_{2}, \gamma_{2}\right)$ be standard related extensions of $B_{1}$ and $B_{2}$, respectively. We wish to show that $C_{1}$ and $C_{2}$ are isomorphic:

We have, in a notation paralleling the above, $B_{i}=\left(A_{i}, R_{i}\right)$, and 
$C_{i}=\left(A_{i}, E_{i}\right)$, where $A_{i}$ is a subalgebra of $B_{i}$ which is mapped isomorphically onto $B_{i} / R_{i}$ by the natural homomorphism of $B_{i}$ onto $B_{i} / R_{i}$. Since $A_{i} \approx B_{i} / R_{i}, \rho$ induces an isomorphism $\lambda$ of $A_{1}$ onto $A_{2}$ and an isomorphism $\mu$ of $T_{1} \approx R_{1} / R_{1}^{2}$ onto $T_{2} \approx R_{2} / R_{2}^{2}$. For $a \in A_{1}, t \in T_{1}$, we shall have $\mu(a t)-\lambda(a) \mu(t) \in E_{2}^{2}$ and $\mu(t a)-\mu(t) \lambda(a) \in E_{2}^{2}$, whence $\mu(a t)=\lambda(a) \mu(t)$ and $\mu(t a)=\mu(t) \lambda(a)$. Evidently, $\mu$ may be extended in a unique fashion to a linear isomorphism $\hat{\mu}$ of $\left(T_{1}, \cdots, T_{1}^{(n)}\right)$ onto $\left(T_{2}, \cdots, T_{2}^{(n)}\right)$, such that, for $t_{j} \in T_{1}$,

$$
\hat{\mu}\left(t_{1} \times \cdots \times t_{k}\right)=\mu\left(t_{1}\right) \times \cdots \times \mu\left(t_{k}\right) .
$$

Clearly, $\hat{\mu}$ maps each $U_{k, 1}$ onto $U_{k, 2}$, where the $U_{k, i}$ are the subspaces of the $T_{i}^{(k)}$, defined as were the $U_{k}$ in our construction above. It follows that $\hat{\mu}$ induces an isomorphism $\bar{\mu}$ of $E_{1}$ onto $E_{2}$, such that $\bar{\mu}(z)-\hat{\mu}(z) \in U_{k, 2}$, for $z \in T_{1}^{(k)}$. From this and the above it follows that $\bar{\mu}(a \cdot e)=\lambda(a) \cdot \bar{\mu}(e)$, and $\bar{\mu}(e \cdot a)=\bar{\mu}(e) \cdot \lambda(a)$, for all $e \in E_{1}$ and $a \in A_{1}$. Finally, the mapping $(a, e) \rightarrow(\lambda(a), \bar{\mu}(e))$ is evidently an isomorphism of $C_{1}$ onto $C_{2}$.

Now let $(C, \gamma)$ be a standard extension of $B$. Let $(D, \delta)$ be any related extension of $C$, and construct a standard related extension $\left(C^{*}, \gamma^{*}\right)$ of $D$. Then, since $D$ and $B$ are related, it follows from what we have just proved that $C^{*}$ is isomorphic with $C$. Hence the homomorphism $\delta \gamma^{*}$ of $C^{*}$ onto $C$ must be an isomorphism. A fortiori, $\delta$ is an isomorphism. Hence $C$ is maximal. This completes the proof of our theorem.

We obtain immediately the following corollary:

Corollary. Every maximal algebra is quasicyclic.

5. Some illustrative remarks. It is interesting that some algebras whose structure one would regard as nondegenerate on purely intuitive grounds are, indeed, quasicyclic or even maximal. Thus, the algebra formed by all $n$ by $n$ matrices with zero coordinates above the main diagonal is maximal. The nilpotent algebra of matrices with zero coordinates on and above the main diagonal is quasicyclic, though evidently not maximal.

A simple example of an algebra which is not quasicyclic is the following: Let $N$ be the maximal nilpotent algebra generated by an element $x$ with $x^{7}=0$, such that the set of powers $\left(x, x^{2}, \cdots, x^{6}\right)$ forms a linear basis for $N$. Let $Z$ be the subalgebra generated by $x^{2}$ and $x^{3}$. Then $Z$ has a linear basis $\left(x^{2}, x^{3}, x^{4}, x^{5}, x^{6}\right), Z^{2}$ has a basis $\left(x^{4}, x^{5}, x^{6}\right)$, while $Z^{3}$ is the one-dimensional vector space spanned by $x^{6}$. If $Z=Z_{1}+Z_{1}^{2}+Z_{1}^{3}$ were a quasicyclic decomposition of $Z$, then $Z_{1}$ would have to contain an element of the form $x^{3}+\alpha x^{4}+\beta x^{5}+\gamma x^{6}$. Then $Z_{1}^{2}$ 
would contain $x^{6}$, that is, $Z_{1}^{2} \supseteq Z^{3}$, which gives a contradiction. Hence $Z$ is not quasicyclic.

6. An application. We shall apply the above methods to a particularly simple case in which a complete result can be obtained.

Let $B$ be an algebra over the field $F$, and let $R$ be the radical of $B$. We consider the case in which $B / R$ is a central simple algebra over $F$, that is, $B / R$ is a full matrix ring of degree $d$ (say) with coordinates in a division algebra $\Phi$ over $F$, such that $F$ is the center of $\Phi$. Then there exists a subalgebra $A$ of $B$ which is mapped isomorphically onto $B / R$ by the natural homomorphism of $B$ onto $B / R$, and $R$ is a twosided $A$-module in the natural fashion. In addition to the above we shall assume that the quotient module $R / R^{2}$ is simple, and that it is not annihilated by $A$ on either side. (This implies, in particular, that the identity element of $A$ acts as the identity transformation in $R / R^{2}$, both on the right and on the left.) Let $\tilde{A}$ be the algebra anti-isomorphic with $A$. Then, by well known results, ${ }^{5} A \times \tilde{A}$ is simple (in fact, it is a full matrix algebra over $F$ ), and, as in the proof of Theorem 2.1, we may regard $R / R^{2}$ as a nontrivial simple left $A \times \tilde{A}$-module. Now the algebra $A$ itself may be regarded in the natural way as such a simple left $A \times \tilde{A}$ module, and since any two nontrivial simple left modules for a simple algebra are isomorphic, we may conclude that there exists an isomorphism $\lambda$ of $R / R^{2}$ onto $A$ (as a vector space over $F$ ) such that $\lambda\{a \cdot \bar{r}\}=a \lambda\{\bar{r}\}$, and $\lambda\{\bar{r} \cdot a\}=\lambda\{\bar{r}\} a$, for every $a \in A$, and every $\bar{r} \in R / R^{2}$. Therefore, in constructing a maximal related extension of $B$ we may take for the space $T$ of $\$ 4$ a copy, $A^{(1)}$, of $A$, and we shall have $a_{1} \cdot a_{2}^{(1)}=\left(a_{1} a_{2}\right)^{(1)} ; a_{2}^{(1)} \cdot a_{1}=\left(a_{2} a_{1}\right)^{(1)}$, where we indicate by $a \rightarrow a^{(1)}$ a fixed isomorphism of $A$ onto $A^{(1)}$.

Next, we shall have to compute the subspace $U_{2}$ of $T \times T$. For this purpose, we may as well use the isomorphic space $A \times A ; U_{2}$ will be the subspace of $A^{(1)} \times A^{(1)}$ which corresponds to the subspace $U_{2}^{\prime}$ of $A \times A$ spanned by all elements of the form $a_{1} a_{2} \times a_{3}-a_{1} \times a_{2} a_{3}$. For a moment, it will be convenient to identify $A$ with $\Phi_{d}$, and to introduce the usual matrix units $e_{i j} ; 1 \leqq(i, j) \leqq d$. Thus, each element of $A$ may be written $a=\sum_{i, j} \phi_{i j} e_{i j}$, with $\phi_{i j} \in \Phi$, and we have $\left(\phi e_{i j}\right)\left(\phi^{\prime} e_{p q}\right)$ $=\delta_{j p} \phi \phi^{\prime} e_{i q}$, where $\delta_{j p}$ stands for the usual Kronecker symbol. Now we have

$$
e_{i j} e_{r s} \times e_{p q}-e_{i j} \times e_{r s} e_{p q}=\delta_{j r} e_{i s} \times e_{p q}-\delta_{s p} e_{i j} \times e_{r q} .
$$

For $j=r$ and $s \neq p$ this gives $e_{i s} \times e_{p q}$, and for $j=r$ and $s=p$ we get $e_{i s} \times e_{s q}-e_{i r} \times e_{r q}$. It follows that every element of $A \times A$ is congruent

5 N. Jacobson, loc. cit. chap. 5, $\$ 13$. 
$\bmod U_{2}^{\prime}$ to a sum of elements of the form $\phi e_{i 1} \times \phi^{\prime} e_{1 j}$. Furthermore, $U_{2}^{\prime}$ contains all elements of the form

$$
\phi_{1} \phi_{2} e_{i 1} \times \phi_{3} e_{1 j}-\phi_{1} e_{i 1} \times \phi_{2} \phi_{3} e_{1 j} .
$$

Take $\phi_{1}=\phi \phi^{\prime}, \phi_{2}=\left(\phi^{\prime}\right)^{-1}, \phi_{3}=\phi^{\prime}$; then we see that $U_{2}^{\prime}$ contains $\phi e_{i 1} \times \phi^{\prime} e_{1 j}-\phi \phi^{\prime} e_{i 1} \times e_{1 j}$. Hence every element of $A \times A$ is congruent $\bmod U_{2}^{\prime}$ to a sum of elements of the form $\phi e_{i 1} \times e_{1 j}$.

Now consider the mapping $a_{1} \times a_{2} \rightarrow a_{1} a_{2}$. This induces a bilinear mapping of $A \times A$ into $A$ which evidently annihilates $U_{2}^{\prime}$. Hence, if $\sum_{i, j} \phi_{i j} e_{i 1} \times e_{1 j} \in U_{2}^{\prime}$, it follows that $\sum_{i, j} \phi_{i j} e_{i j}=0$, that is, $\phi_{i j}=0$. Therefore, the above mapping induces an isomorphism of $A \times A / U_{2}^{\prime}$ onto $A$. Since $\left(\phi e_{i 1} \times e_{1 j}\right) \cdot \phi^{\prime} e_{p q}=\phi e_{i 1} \times \phi^{\prime} e_{1 j} e_{p q} \equiv \phi \phi^{\prime} e_{i 1} \times e_{1 j} e_{p q}\left(U_{2}^{\prime}\right)$, and similar relations hold for the left operations, this isomorphism is actually an operator isomorphism. Therefore, we may identify $V_{2}$ (cf. \$4) with a copy, $A^{(2)}$, of $A$, with the same relations as we had obtained above for $T$ and $A^{(1)}$. Almost identical computations show that each $V_{k}$ may be identified with a copy $A^{(k)}$ of $A$.

It follows that, if $(C, \gamma)$ is a maximal related extension of $B, C$ is isomorphic with the algebra obtained as follows : Let $A^{(0)}, A^{(1)}, \cdots, A^{(n)}$ be $(n+1)$ copies of $A$, where $n$ is the index of nilpotency of $R$. Define $a_{1}^{(r)} \circ a_{2}^{(s)}=\left(a_{1} a_{2}\right)^{(r+s)}$, for $r+s \leqq n$, and 0 otherwise, and extend this by linearity to give the structure of an algebra over the direct sum of the vector spaces, $A^{(0)}, \cdots, A^{(n)}$. (Evidently, the radical of $C$ is $A^{(1)}+\cdots+A^{(n)}$, and we have $A^{(k)}=\left(A^{(1)}\right)^{k}$.) A still simpler description of this algebra, $C$, is obtained by noting that $C$ can be obtained from the polynomial ring $A[x]$ by dividing out the two-sided ideal generated by $x^{n+1}$.

Now let $I$ be any nonzero two-sided ideal which is contained in the radical $A^{(1)}+\cdots+A^{(n)}$. Then the projections $I^{(k)}$ of $I$ onto the $A^{(k)}$ are evidently submodules of the $A$-module $A^{(1)}+\cdots+A^{(n)}$. Let $k_{0}$ be the least $k$ for which $I^{(k)} \neq\{0\}$. Since $A^{\left(k_{0}\right)}$ is a simple module we must have $I^{\left(k_{0}\right)}=A^{\left(k_{0}\right)}$. Now $A^{\left(n-k_{0}\right)} \circ I^{\left(k_{0}\right)} \subseteq I$. But $A^{\left(n-k_{0}\right)} \circ I^{\left(k_{0}\right)}=A^{(n)}$. Hence we have $I \supseteq A^{(n)}$. We may now consider $I / A^{(n)}$ in $C / A^{(n)}$ and conclude that $I \supseteq A^{(n-1)}$, unless $k_{0}=n$, and so on. Finally, we conclude that $I=A^{\left(k_{0}\right)}+\cdots+A^{(n)}$. But this shows that the homomorphism $\gamma$ of $C$ onto $B$ must be an isomorphism, for otherwise we could take for $I$ the kernel of $\gamma$ and conclude that $B \approx C / A^{\left(k_{0}\right)}$, which contradicts the assumption that the index of nilpotency of $R$ is $n$. Hence every algebra such as $B$ is maximal and has the same simple structure as the algebra $C$ which we have constructed.

In the general case, it is not difficult to construct the maximal algebras, starting from a decomposition of the $A$-module $R / R^{2}$ into a di- 
rect sum of simple submodules. The real difficulties are met in the attempt to enumerate the two-sided ideals which are contained in the radical.

HARVARD UNIVERSITY

\section{ON A CONJECTURE ABOUT INFINITE CLASS FIELDS}

\section{GEORGE WHAPLES}

If we are given any algebraic extension field, of finite degree, of a given ground field, then the $p$-adic completion of the extension field, under any one of its valuations ${ }^{1}$ (prime spots) is an algebraic extension of the completion of the ground field under the same valuation. Our original extension field (in the large) thus determines a set of algebraic extensions of $p$-adic ground fields. We shall refer to these extensions as the local components of the original field. If our extension field (in the large) is normal, then any two valuations of the extension field which induce the same valuation in the ground field determine isomorphic local components; hence in case of a normal extension field we can think of a local component as determined by a valuation of the ground field.

When our extension field is not of finite degree we must modify this definition, since the $p$-adic closure of such an extension field will in general not be algebraic over the ground field. ${ }^{2}$ For a normal extension of infinite degree we define the local component as follows: The original extension is the splitting field ${ }^{3}$ of a certain set of polynomials with coefficients in the ground field. Define the local component to be the splitting field of this same set of polynomials over the $p$-adic extension of the ground field. It is easy to show that this field is independent of the set of polynomials used (indeed, one could use the set of all polynomials of the ground field which split in the extension

Received by the editors August 12, 1946, and, in revised form, November 13, 1946.

${ }^{1}$ For theory of valuations, see E. Artin and G. Whaples, Axiomatic characterization of fields by the product formula for valuations, Bull. Amer. Math. Soc. vol. 51 (1945) pp. 469-492, and the literature cited there.

2 See Ostrowski, Über einige Fragen der allgemeine Körpertheorie, Journal für Mathematik vol. 143 (1914) pp. 225-284. I am indebted to the referee for a correction of the first version of this paper and for this reference.

${ }^{3}$ The splitting field is the smallest subfield of the algebraic closure in which all the given polynomials split into linear factors. 